\title{
Le salariat agricole. Une part croissante dans l'emploi des exploitations mais une précarité des statuts
}

Éric Cahuzac et Cécile Détang-Dessendre

\section{(2) OpenEdition \\ 1 Journals}

\section{Édition électronique}

URL : http://journals.openedition.org/economierurale/3050

DOI : $10.4000 /$ economierurale.3050

ISSN : 2105-2581

Éditeur

Société Française d'Économie Rurale (SFER)

\section{Édition imprimée}

Date de publication : 23 mai 2011

Pagination : 82-92

ISSN : 0013-0559

\section{Référence électronique}

Éric Cahuzac et Cécile Détang-Dessendre, «Le salariat agricole. Une part croissante dans l'emploi des exploitations mais une précarité des statuts », Économie rurale [En ligne], 323 | mai-juin 2011, mis en ligne le 23 mai 2013, consulté le 30 avril 2019. URL : http://journals.openedition.org/ economierurale/3050; DOI : 10.4000/economierurale.3050 
FAITS ET CHIFFRES

\title{
Le salariat agricole Une part croissante dans l'emploi des exploitations mais une précarité des statuts
}

\author{
Éric CAHUZAC・INRA-ODR, Toulouse \\ Cécile DÉTANG-DESSENDRE・INRA-CESAER, Dijon
}

Cette étude propose un panorama de la situation de l'emploi salarié agricole en France et de son évolution au cours de la période contemporaine en mobilisant différentes sources statistiques. Après avoir décrit les conditions générales de l'emploi salarié - le type de contrat, la nature de l'employeur - les auteurs précisent les caractéristiques de la main-d'œuvre, en comparant cette dernière à celle des autres secteurs. Les niveaux de rémunération font enfin l'objet d'une attention particulière.

n France, le recul du nombre d'exploitaEtions agricoles se poursuit inexorablement (cf. l'encadré méthodologique). Sur près de vingt ans, la moitié des exploitations et des actifs ont disparu en agriculture avec des taux de décroissance voisins de $2,5 \%$ en moyenne par an et qui s'accentuent ces dernières années. En 2007, avec 3,5 \% de la population active, l'agriculture assure un emploi à près d'un million de personnes (tableau 1). Plus des trois quarts de ces actifs sont concentrés dans les exploitations professionnelles (voir lexique, in fine) qui occupent chacune en moyenne près de 2,4 travailleurs, sous des formes différentes. Les estimations faites à l'horizon de cinq à dix ans conver- gent vers une baisse continue du nombre d'exploitations. Ainsi, selon Piet $(2008)^{1}$ ou Fall et ali $(2010)^{2}$, qui s'appuient sur les données du RICA3, 250000 exploitations

1. Piet L. (2008). La démographie agricole française : combien d'exploitations en 2015 ? Série Alimentation-Agriculture-Environnement, numéro spécial Emplois agricoles et ruraux.

2. Fall M., Piet L., Roger M. (2010). L'évolution de la population des exploitations agricoles professionnelles françaises. Revue d'Études en agriculture et environnement, 91/3, p. 279-295.

3. Réseau d'information comptable agricole; source Service de la statistique et de la prospective (SSP) du ministère de l'Agriculture, de l'Alimentation et de la Pêche.

Tableau 1. Évolution de la structure des exploitations agricoles

\begin{tabular}{l|c|c|c|c}
\hline \multirow{2}{*}{ Nombre d'exploitations } & \multicolumn{4}{|c}{ France métropolitaine (millier) } \\
\cline { 2 - 5 } & 1988 & 2000 & 2005 & 2007 \\
\hline Actifs totaux & 1016,8 & 663,8 & 545,3 & 506,9 \\
\hline Nombre d'exploitations professionnelles & 2031 & 1313 & 1100 & 1020 \\
\hline Actifs des exploitations professionnelles & 608,5 & 393,9 & 346,5 & 326,2 \\
\hline SAU moyenne des exploitations professionnelles (ha) & 1458 & 938 & 822 & 769 \\
\hline
\end{tabular}

Source : Agreste-SSP - Enquêtes structure 2005 et 2007, recensement agricole 2000 (même échantillon); pour 1988, SCEES recensement agricole.

Note : le nombre d'actifs sur l'exploitation comprend ici : les chefs d'exploitations et les co-exploitants, les conjoints non co-exploitants, les autres actifs familiaux ainsi que les salariés permanents non familiaux. 


\section{Encadré méthodologique. Les sources statistiques utilisées}

Pour réaliser cette étude, différentes sources sont mobilisées.

Enquêtes structures : réalisées par le service de la statistique et de la prospective (SSP) du ministère de l'Agriculture, de l'Alimentation, et de la Pêche. Ces enquêtes portent sur des exploitations agricoles identifiées lors du dernier recensement agricole (RA) et sont menées par sondage stratifié durant les périodes intercensitaires. Ces enquêtes permettent d'actualiser les résultats des recensements réalisés tous les dix ans en moyenne. Elles permettent de suivre la population agricole, de connaître l'évolution des productions agricoles et de la structure des exploitations. Les dernières enquêtes Structures ont été réalisées en 2003, 2005 et 2007.

Enquête emploi : réalisée par l'Insee, cette enquête vise à observer à la fois de manière structurelle et conjoncturelle la situation des personnes vis-à-vis du marché du travail. Annuelle jusqu'en 2002, l'enquête emploi est trimestrielle et sa collecte est réalisée en continu depuis 2003. L'échantillonnage est aréolaire' sur une base d'environ 45000 logements. Toutes les personnes de 15 ans et plus résidant dans le logement enquêté sont interrogées. Cette source permet de comparer les salariés de l'agriculture avec les agriculteurs mais aussi avec les salariés des autres branches d'activité. Elle renseigne aussi sur les niveaux de revenus. Il faut pour autant garder en tête qu'il s'agit d'une enquête et que les résultats comportent une certaine part d'aléa (Insee, 2008) ${ }^{2}$, en particulier, les salariés agricoles enquêtés sont peu nombreux, un peu plus de 1300.

Fichiers des cotisants : cette source provient de la Mutualité sociale agricole (MSA) et regroupe tous les chefs d'exploitation en activité cotisant à l'une ou à l'ensemble des trois branches : maladie, prestation familiale, vieillesse. Contrairement aux enquêtes, ces données sont exhaustives sur leur champ. Cette étude a mobilisé également des données agrégées provenant d'Agreste ou d'Eurostat ainsi que celles des tableaux de bord salariés et non salariés de la MSA. Pour ce qui concerne les statistiques Agreste (SSP), sauf indication contraire, le champ retenu est celui des exploitations professionnelles de France métropolitaine.

Compte tenu de la diversité des concepts mobilisés par les différentes sources (exploitation, chef d'exploitation, cotisant, contrat de travail...), les résultats obtenus ne sont pas directement comparables. Les effectifs et évolutions sont donc analysés par source et seuls les ordres de grandeur et les grandes tendances peuvent être rapprochés. L'emploi tel qu'il est mesuré ici est restreint à celui des activités de production des exploitations agricoles. Dans les sections 2 (l'emploi salarié) et 3 (Travail familial/salarié) qui utilisent l'enquête emploi, on ne prendra donc pas en compte les emplois liés à la transformation des biens agricoles ou alimentaires, ni les emplois dans les services (paysagiste, espaces verts...).

1. Méthode qui consiste à prélever l'échantillon de la population visée en partant de sa répartition géographique. 2. Insee (2008). Enquête, emploi en continu (à partir de 2003). Sources et méthodes.

professionnelles subsisteraient à l'horizon 2015, tandis que Butault et Delame (2007) ${ }^{4}$, en projetant l'enquête SSP sur la structure des exploitations agricoles de 2005, estiment ce chiffre à 270000 pour la même date et n'avancent qu'une estimation de 200000 exploitations professionnelles pour 2030. Dans une autre étude s'appuyant sur les données de la Mutualité sociale agricole

4. Butault J.-P., Delame N. (2007). Combien d'exploitations en 2015 ? Rapport pour la prospective Agriculture 2013.
(MSA), Lefèbvre (2009) ${ }^{5}$ estime le nombre de chefs d'exploitation à 320000 en 2020 soit une diminution de plus d'un quart du nombre actuel de chefs selon cette même source. Un aperçu de ce changement structurel est donné dans le tableau 1.

5. Lefèbvre M.-F. (2009). Démographie agricole : la France doit-elle craindre l'avenir? Déméter. 


\section{Des changements structurels s'opèrent au sein des exploitations}

Les disparitions d'exploitations libèrent environ 1 million d'ha en moyenne par an, qui sont utilisés pour près de moitié à l'accueil de nouveaux agriculteurs ${ }^{6}$, tandis que $40 \%$ bénéficient à l'agrandissement des exploitations existantes, $10 \%$ sont artificialisés sous l'effet de l'urbanisation et de l'étalement urbain, et $3 \%$ convertis en forêt ${ }^{7}$.

En conséquence, la surface moyenne des exploitations augmente : en 2007, les exploitations professionnelles ont en moyenne une surface agricole utile de 77 ha (42 ha en 1988) tandis qu'elle est de 54 ha en moyenne pour l'ensemble des exploitations. Ce phénomène de concentration est en partie à l'origine de changements structurels. Quelles qu'en soient les raisons (démographiques, techniques ou économiques), cette augmentation de la taille des exploitations exige plus de moyens et d'immobilisations, et va s'accompagner d'une modification de leurs statuts juridiques ${ }^{8}$.

6. Lefèbvre (op. cit.) estime à 16000 le nombre d'installations par an en moyenne (depuis 2000). Ces installations se font sur des superficies de 36 ha en moyenne.

7. Voir l'Inventaire forestier national pour plus d'information sur les surfaces forestières en France. 8. Cette modification juridique répond aussi à une volonté politique, celle de vouloir séparer le patrimoine de l'agriculteur de celui de l'exploitation, initiée avec la création des GAEC (Groupements agricoles d'exploitation en commun) en 1962, puis des EARL en 1965 (Entreprises agricoles à responsabilité limitée). Marginales au début, les sociétés se sont fortement développées à partir de 2000, pour représenter actuellement, tous statuts confondus, $40 \%$ des exploitations professionnelles.
Mais c'est sur l'évolution de la structure de la main-d'œuvre que les transformations sont les plus intenses, notamment pour le salariat, lequel concerne, en 2007, une exploitation professionnelle sur deux.

\section{L'emploi salarié}

Sous des formes diverses, l'emploi salarié est présent dans près d'une exploitation sur deux. Dans le mouvement général de recul du nombre d'actifs sur les exploitations agricoles entre 1988 et 2007, la baisse du nombre de salariés a été moins forte que celle des actifs familiaux et, de fait, la part du salariat dans la main-d'œuvre de l'exploitation a progressé sur cette période (Blanc et al., 2008) ${ }^{9}$. Ainsi, en 2000, l'emploi salarié agricole représentait près de 242000 équivalents temps plein et 230000 en 2007, soit une baisse de $5 \%$ des effectifs (tableau 2). Dans la même période, les actifs familiaux non salariés enregistraient quant à eux une baisse de $19 \%$ de leur effectif. En 2007 , près de $32 \%$ des heures travaillées sur l'exploitation (UTA) ${ }^{10}$ sont réalisées par des salariés contre seulement $19 \%$ en 1988.

Cette progression forte de la part des UTA salariées dans les UTA totales est un phénomène général qui se retrouve au

9. Blanc M., Cahuzac E., Elyakime B., Tahar G. (2008). Demand for on-farm permanent hired labour on family holdings. European Revue of Agricultural Economics, 35, p. 493-518.

10. Unité de travail annuel : quantité de travail annuel d'une personne à temps plein.

Tableau 2. Évolution du travail sur les exploitations agricoles selon le statut

\begin{tabular}{l|r|r|r|c}
\hline & \multicolumn{4}{|c}{ France métropolitaine (millier) } \\
\cline { 2 - 5 } & 1988 & 2000 & 2005 & 2007 \\
\hline Actifs familiaux & 1010 & 612 & 536 & 500 \\
\hline dont actifs familiaux salariés & 14 & 20 & 21 & 22 \\
\hline Salariés permanents non familiaux & 138 & 126 & 120 & 118 \\
\hline Salariés saisonniers & \multirow{2}{*}{86} & 89 & 87 & 82 \\
\cline { 1 - 3 } Salariés des ETA et CUMA & & 6,9 & 8,0 & 8,6 \\
\hline
\end{tabular}

Source : Agreste-SSP - Enquêtes structure 2005 et 2007, recensement agricole 2000 (même échantillon); pour 1988, SCEES recensement agricole. 
FAITS ET CHIFFRES

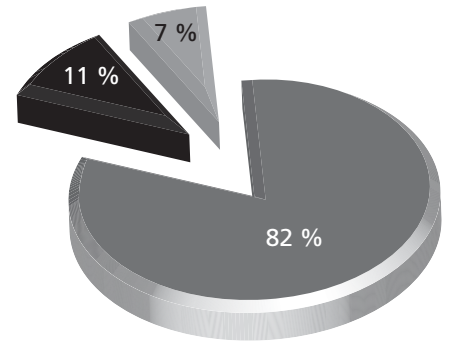

Salariés saisonniers

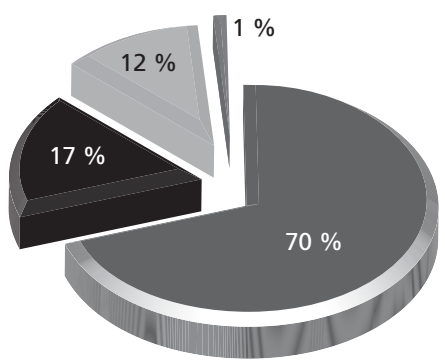

Actifs familiaux
Salariés des ETA et CUMA

ETA : Entreprise de travaux agricoles • CUMA : Coopérative d'utilisation de matériel agricole

Source : Agreste-SSP - Enquêtes structure 2007. Pour 1988, SCEES recensement agricole.

niveau de l'Union européenne. Au début des années 1990, la proportion était de $14 \%$, actuellement les salariés fournissent en moyenne un quart de la main-d'œuvre agricole. Dans les grandes exploitations européennes (supérieures à 150 ha équivalent blé), la main-d'œuvre salariée représente même près des deux tiers (64\%) de la maind'œuvre totale, un chiffre quasi identique à celui de la France.

\section{Une substitution progressive entre travail familial et travail salarié}

Le nombre d'actifs familiaux qui adoptent le statut de salarié a augmenté de $36 \%$ entre 1988 et 2007 . Ce statut reste très minoritaire dans le travail familial agricole (moins de $5 \%$ des effectifs). Cependant, ces salariés issus des familles agricoles représentent près de $10 \%$ des salariés. Au-delà de cette évolution marginale de statut des actifs familiaux, le salariat non familial, qui se substitue peu à peu à la main-d'œuvre familiale sur l'exploitation, peut être de nature fort diverse. Il peut être permanent, c'est-à-dire relatif à des personnes embauchées à temps complet ou partiel, mais travaillant de façon régulière sur l'exploitation (toutes les semaines ou tous les mois). Ce type de main-d'œuvre reste proportionnellement majoritaire sur l'exploitation et représente $56 \%$ des équivalents temps plein salariés non familiaux en 2007. Cette proportion reste constante entre 2000 et 2007 . Ces salariés sont principalement présents sur des activités de maraîchage, d'horticulture et de viticulture (figure 1).

Le salariat est aussi saisonnier, lorsqu'il correspond à des contrats de travail d'une durée inférieure à six mois (à temps plein ou partiel). Plus des trois quarts de cette maind'œuvre saisonnière (78 \%) sont employés dans les secteurs de la viticulture, de l'arboriculture, des grandes cultures et des cultures maraîchères. En 2007, près de 40 \% des exploitations professionnelles utilisent de la main-d'œuvre saisonnière (Agreste-Structure, 2008) ${ }^{11}$ et cette part est en augmentation constante depuis 2000, année où elle représentait seulement $34 \%$. Ce phénomène est lié à des taux de survie différents au sein des exploitations professionnelles.

En effet, alors que le nombre d'exploitations professionnelles diminue de $17 \%$ entre 2000 et 2007 , le nombre d'exploitations utilisant de la main-d'œuvre salariée saisonnière diminue seulement de $6 \%$. C'est parmi les exploitations sans salarié que les

11. AGRESTE (2008). Enquête sur la structure des exploitations professionnelles en 2007, 326000 exploitations professionnelles. Primeur, $\mathrm{n}^{\circ} 215$, octobre. 
disparitions ont été les plus nombreuses sur cette période ( $25 \%$ en moins). Malgré tout, en équivalent temps plein, la part des saisonniers dans la main-d'œuvre salariée (non familiale) sur l'exploitation reste à un niveau constant depuis 2000 (41\%).

Enfin, le salariat peut être partagé et les exploitations peuvent avoir recours à des Entreprises de travaux agricoles (ETA), à des Coopératives d'utilisation de matériel agricole (CUMA) ainsi qu'à des Groupements d'employeurs (GE) ${ }^{12}$. En 2005, l'emploi partagé représente un total de plus de 90000 salariés (ANEFA, 2005) ${ }^{13}$. Les ETA et CUMA représentent environ $1 \%$ de la totalité des heures travaillées sur l'exploitation, mais $60 \%$ des exploitations font appel à de tels emplois, principalement dans les secteurs de la viticulture, de l'élevage et des grandes cultures. L'emploi salarié dans les CUMA est, quant à lui, modeste (6 500 salariés en 2005, moins de 2000 UTA), ces

12. Une difficulté réside dans l'identification de ces différents types d'emploi à partir des statistiques nationales. En effet, ETA et CUMA sont agrégés tandis que les GE sont comptabilisés avec les salariés permanents dans les statistiques Agreste et regroupés avec les services de remplacement. En 2005 les services de remplacement comptent 2600 ETP selon l'Observatoire de l'emploi salarié en agriculture de l'ANEFA.

13. ANEFA (2005). Regards sur l'emploi salarié en agriculture. structures ayant pour but principal de mettre à disposition des exploitants le matériel agricole qui leur est nécessaire.

\section{Développement des groupements d'employeurs sans stabilisation de la main-d'œuvre}

Les Groupements d'employeurs (GE) se sont quant à eux développés ces dernières années pour faire face en partie à la demande - non satisfaite par les ETA et les CUMA de travail permanent à temps partiel. Ce sont principalement les petites exploitations avec un besoin de travail salarié toute l'année mais en petite quantité qui sont demandeuses, en premier lieu dans l'élevage et les grandes cultures. On comptait en 1995 environ un millier de groupements, ils sont près de quatre fois plus nombreux en 2007 (3 700) selon la MSA. Ce sont 60000 contrats et 16000 Emplois à temps plein (ETP) qui contribuent, en 2007, à l'effort de production des exploitations agricoles en France. L'emploi en contrat à durée déterminée prédomine dans les groupements d'employeurs ainsi que l'emploi à temps partiel. En effet, Elyakime (2007) ${ }^{14}$ montre que plus de $75 \%$ des emplois des GE sont

14. Elyakime B. (2007). Groupements d'employeurs agricoles : quelle aide publique locale ? Revue d'économie régionale et urbaine, 5, p. 861-880.

Figure 2. Évolution des contrats de groupements d'employeurs

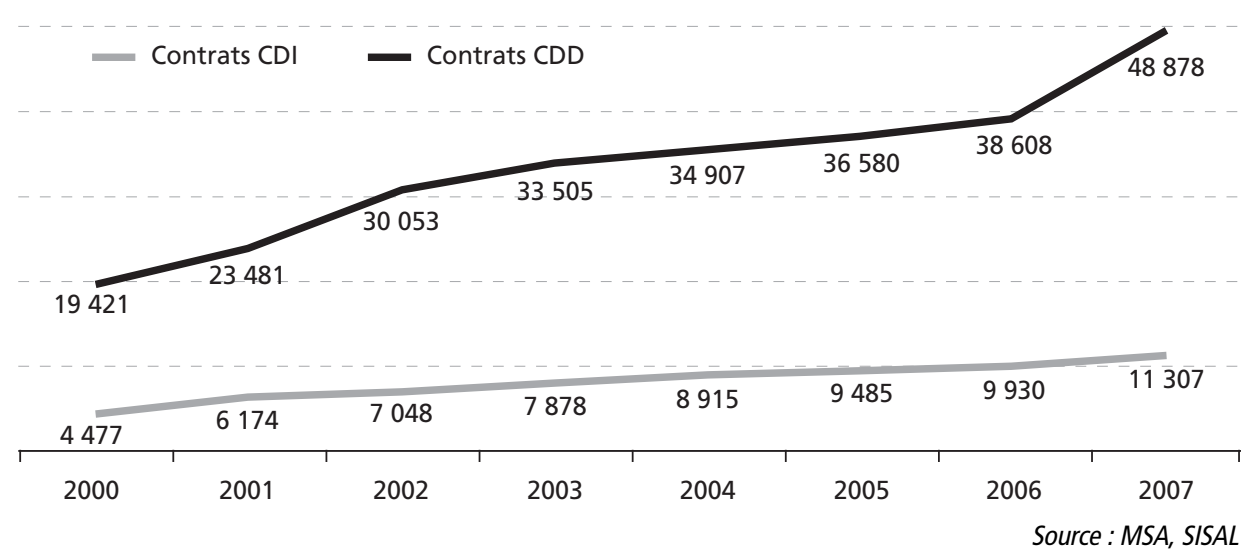


FAITS ET CHIFFRES

Éric CAHUZAC, Cécile DÉTANG-DESSENDRE

Figure 3. Cartes de répartition régionale de la part des salariés et des saisonniers
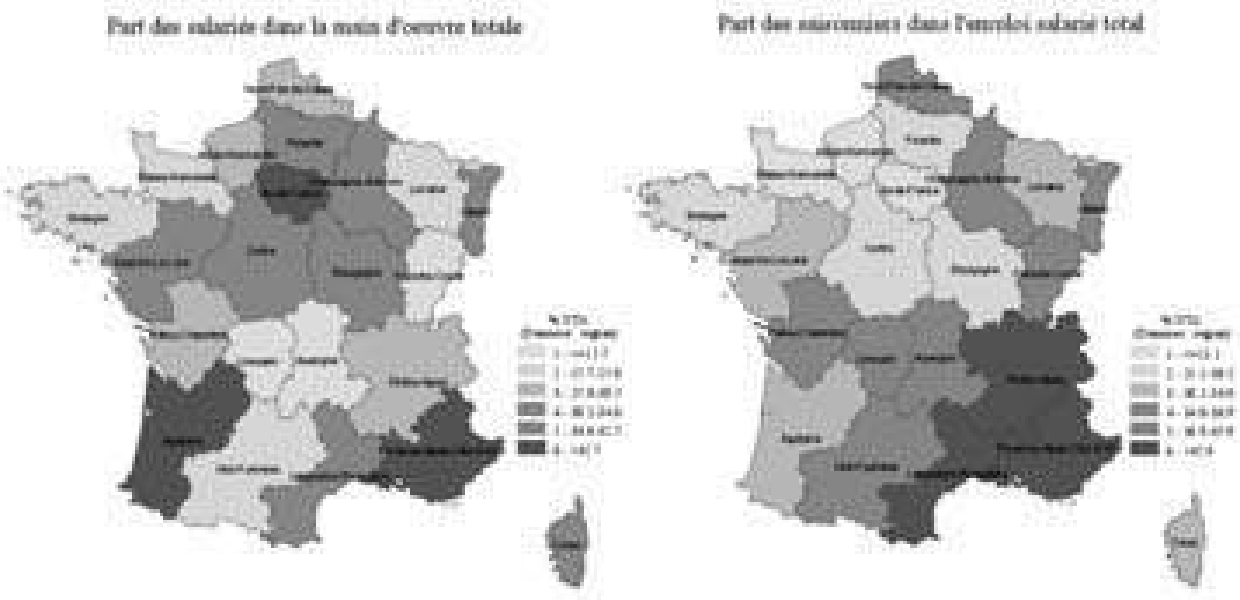

Source : Agreste-SSP - Enquêtes structure 2007, traitements INRA-ODR.

à temps partiel (CDI, saisonniers ou occasionnels) en 2003. De même, selon la MSA, $80 \%$ des contrats de salariés de groupement sont à durée déterminée en 2007 comme le montre la figure 2.

À l'origine (loi du 25 juillet 1985) constitué dans le but exclusif de mettre à disposition des entreprises de moins de dix salariés - membres du groupement d'employeurs - la main-d'œuvre salariée nécessaire, les mutations législatives et réglementaires qu'ont connues les groupements d'employeurs ont fait de cet outil un instrument de pilotage de la main-d'œuvre salariée en agriculture. Les groupements se sont peu à peu ouverts aux collectivités locales et à des entreprises adhérentes de taille plus importante (plus de 300 salariés depuis 2000), permettant ainsi aux entre- prises qui utilisent de la main-d'œuvre en quantité de bénéficier d'un vivier de salariés dans tous les domaines d'activités (figure 3).

La dimension collective du groupement d'employeurs avait aussi pour objectif la stabilité de l'emploi ainsi créé. Même si cette stabilité reste à l'ordre du jour vingt ans après, dans les faits, l'emploi à durée déterminée prédomine ainsi que le temps partiel, induisant des changements d'activités nombreux pour les salariés concernés.

\section{Une main-d'œuvre salariée peu formée, masculine et plutôt jeune}

La description de la main-d'œuvre salariée agricole qui va suivre s'appuie sur l'exploitation de l'Enquête emploi de 2005

Tableau 3. Répartition des actifs agricoles par niveau de diplôme selon le statut (en \%)

\begin{tabular}{l|c|c|c|c|c|c}
\hline \multicolumn{2}{|c|}{} & \multicolumn{4}{c}{ Salariés } \\
\hline & $\begin{array}{c}\text { Population } \\
\text { active }\end{array}$ & $\begin{array}{c}\text { Agriculteurs } \\
\text { exploitants }\end{array}$ & Agriculture & IAA & Autres ind. & Commerce \\
\hline Diplôme supérieur & 29,3 & 11,6 & 8,7 & 12,4 & 22,6 & 19,79 \\
\hline Bac ou brevet prof & 17,2 & 17,8 & 18,1 & 14,1 & 14,9 & 21,12 \\
\hline CAP, BEP & 26,7 & 38,8 & 29,9 & 39,5 & 33,7 & 32,17 \\
\hline Brevet, CEP, aucun & 26,3 & 31,8 & 43,3 & 34 & 28,8 & 26,92 \\
\hline
\end{tabular}


Tableau 4. Répartition des emplois salariés par catégorie socioprofessionnelle selon le secteur

\begin{tabular}{l|c|c|c|c|c}
\hline & $\begin{array}{c}\text { Ensemble } \\
\text { salariés }\end{array}$ & Agriculture & IAA & Autres ind. & Commerce \\
\hline Cadres & 16,2 & 2,7 & 4,9 & 14,9 & 9,9 \\
\hline Professions intermédiaires & 25,4 & 9,0 & 15,5 & 25,3 & 22,2 \\
\hline Employés & 32,0 & 6,2 & 16,0 & 6,9 & 43,4 \\
\hline Ouvriers & 26,4 & 82,1 & 63,5 & 52,9 & 24,5 \\
\hline
\end{tabular}

Source : Enquête emploi 2005, traitements Inra

de l'Insee (voir encadré méthodologique). Les salariés du secteur agricole sont très majoritairement des hommes $(70 \%)$. Cette distribution est très proche de celle observée dans le secteur industriel, les femmes étant plus employées dans le secteur des services. L'âge moyen des chefs d'exploitation est supérieur de 7 ans à l'âge moyen de la population active française (40 ans), et plus de quatre agriculteurs sur dix ont plus de 50 ans. En revanche, l'âge moyen des salariés du secteur agricole n'est que de 37,5 ans, inférieur de 2,5 ans à l'âge moyen de la population active française et moins de deux salariés agricoles sur dix ont plus de 50 ans.

Ces hommes, plutôt jeunes, salariés du secteur agricole, sont aussi très faiblement formés (tableau 3). Les salariés sans formation sont surreprésentés (plus de $43 \%$ sont sortis du système de formation avec au mieux un brevet des collèges) par rapport à la distribution des niveaux de formation dans la population active totale. La part des salariés diplômés de l'enseignement supérieur dans les exploitations agricoles est quant à elle trois fois plus faible que celle observée dans la population active française dans son ensemble. Ce niveau de formation moyen-faible est à mettre en relation avec le type d'emploi proposé, très majoritairement d'exécution. Les exploitants agricoles, quant à eux, certainement sous la pression des règles qui encadrent l'installation, sont surreprésentés dans les formations techniques de niveau CAP-BEP et baccalauréat professionnel.

Dans le secteur agricole, comme dans l'ensemble de la population active, les femmes sont, plus souvent que les hommes, diplômées de l'enseignement supérieur. Pour autant, l'observation des niveaux de formation par genre et secteur met en évidence que la sous-représentation des diplômés du supérieur en agriculture est comparable pour les hommes et les femmes.

\section{Des emplois salariés moins stables et moins qualifiés que dans les autres secteurs}

Les emplois du secteur agricole sont très majoritairement peu qualifiés : plus de quatre emplois sur cinq sont des emplois d'ouvrier (tableau 4). Pour comparaison, les ouvriers ne représentent qu'un emploi sur quatre dans l'ensemble de la population active et un peu plus d'un sur deux dans le secteur industriel. En corollaire, les postes d'encadrement ne pèsent rien (moins de $3 \%$ ) et, lorsqu'on ajoute les postes de cadres et de professions intermédiaires (essentiellement technicien dans le secteur agricole), l'ensemble ne représente guère plus d'un emploi sur dix, contre plus de quatre sur dix dans la population active totale. La part des postes d'encadrement dans le secteur industriel hors Industries agroalimentaires (IAA) est comparable à celle observée tous secteurs confondus, mais les IAA se distinguent avec un taux d'encadrement divisé par deux.

La comparaison des niveaux de formation des ouvriers du secteur agricole avec ceux de l'industrie et du commerce permet d'affiner la comparaison. Les ouvriers agricoles ont plus souvent que les autres ouvriers de très faibles niveaux de formation : près de la moitié sont sortis du système de formation 
avec au plus le brevet des collèges contre 35 à $40 \%$ dans les deux autres secteurs. En revanche, ils sont plus souvent de niveau bac et bac +2 que les ouvriers de l'industrie ou du commerce (19\% contre moins de $15 \%$ ). Ce dernier élément laisse supposer que les salariés agricoles sont généralement déclarés ouvriers, quel que soit leur type d'emploi, qui peut être un emploi technique nécessitant un niveau de formation plus élevé.

Les salariés agricoles, moins que les salariés des autres secteurs, accèdent à des contrats à durée indéterminée : $74 \%$ contre $85 \%$ sur l'ensemble des actifs salariés en 2005 (tableau 5). C'est le secteur industriel qui offre la plus grande stabilité à ses salariés avec $90 \%$ de contrats à durée indéterminée. Si la proportion de contrats à durée déterminée est comparable dans le secteur agricole et dans l'ensemble des secteurs, c'est bien la place importante des emplois saisonniers qui caractérise la précarité de l'emploi agricole, avec près de $12 \%$ des emplois. Les femmes sont tout particulièrement concernées par l'instabilité en agriculture et par les emplois saisonniers. Dans le commerce ou dans l'industrie, la sousreprésentation des femmes dans les CDI est finalement faible et ne dépasse pas 2 à 3 points de pourcentage. Dans le secteur agricole, en revanche, $62 \%$ des femmes ont un CDI pour plus de $74 \%$ des hommes. Elles représentent plus de $55 \%$ des emplois saisonniers alors qu'elles constituent moins de $30 \%$ de la main-d'œuvre agricole.

Comme pour l'ensemble des salariés français, la stabilité professionnelle des salariés agricoles croît avec l'âge sans pour autant augmenter au même rythme, ni atteindre le même niveau. Entre 25 et 30 ans, la part des CDD est assez comparable, quel que soit le secteur d'activité, et avoisine les $80 \%$. C'est entre 30 et 35 ans que le fossé se creuse : dans l'industrie comme dans le commerce, plus de $90 \%$ des salariés bénéficient d'un CDI en 2005 et cette proportion reste stable quand l'âge avance ; dans l'agriculture, la barre des $80 \%$ de CDI est atteinte autour de 40 ans et cette part ne progresse plus ensuite.

La part de travail à temps partiel est très différente selon les secteurs d'activité : très faible dans l'industrie, elle atteint plus de $20 \%$ dans le commerce. En agriculture, cette part est proche de la moyenne nationale, autour de $15 \%$. Quel que soit le secteur, les femmes travaillent plus souvent à temps partiel que les hommes. Près de $30 \%$ des ouvrières de l'agriculture ne travaillent pas à temps plein, c'est le cas de plus de $40 \%$ des employées de commerce et de seulement $11 \%$ des ouvrières de l'industrie.

\section{Des rémunérations faibles et peu dispersées}

L'enquête emploi fournit différentes informations sur les niveaux de revenu. Nous nous appuyons ici sur les revenus mensuels nets correspondants à des emplois à plein temps redressés des nonréponses et tenant compte des primes. En 2005, la rémunération moyenne perçue par les salariés agricoles avoisine 1200 euros et est inférieure de près de

Tableau 5. Répartition des emplois salariés par type de contrat selon le secteur

\begin{tabular}{l|c|c|c|c|c}
\hline & $\begin{array}{c}\text { Ensemble } \\
\text { salariés }\end{array}$ & Agriculture & IAA & $\begin{array}{c}\text { Autres } \\
\text { industries }\end{array}$ & Commerce \\
\hline Contrats à durée indéterminée & 85 & 74,3 & 87,4 & 89,9 & 89,4 \\
\hline Contrats à durée déterminée & 11 & 12,1 & 5,8 & 4 & 7,3 \\
\hline Saisonniers & 0,8 & 12 & 1,2 & 0,1 & 1,6 \\
\hline Intérim & 3,2 & 1,6 & 5,6 & 6 & 1,7 \\
\hline
\end{tabular}

Source : Enquête emploi 2005, traitements Inra. Les calculs sont réalisés hors contrat d'apprentissage. 
$32 \%$ à la rémunération moyenne des salariés français tous secteurs confondus, laquelle est supérieure à 1700 euros (tableau 6). Cette dernière est tirée vers le haut par les salaires versés dans le tertiaire supérieur (services aux entreprises, banques-assurances...). Les emplois du secteur agricole sont surtout des emplois d'exécution et la faible qualification de ces emplois se traduit logiquement par un niveau de salaire moyen-faible, un salaire global moyen proche de celui des ouvriers et une très faible proportion de salariés dépassant 1500 euros : ils sont moins de $13 \%$ en agriculture contre plus de $50 \%$ tous secteurs confondus.

\section{Des salaires des ouvriers agricoles proches de ceux des employés de commerce}

La comparaison des niveaux de rémunération perçus par les ouvriers selon le secteur d'activité montre que la prise en compte des différences de structure sectorielle des qualifications des emplois n'explique pas toute la sous-rémunération des salariés du secteur agricole ( $c f$. tableau 5). En effet, les ouvriers agricoles perçoivent une rémunération de moins de 1100 euros, inférieure en moyenne de $20 \%$ à la rémunération moyenne d'un ouvrier de l'industrie en France.

Il faut regarder du côté des employés du commerce pour trouver une distribution des rémunérations proche de celle des ouvriers agricoles : le salaire médian est comparable, mais le salaire moyen et le dernier décile sont supérieurs de $10 \%$ dans le commerce. Si la grande majorité des salariés du commerce et de l'agriculture ont des salaires faibles et proches du SMIC, pour autant, la frange supérieure des salaires dans les emplois de commerce, mieux rémunérés, tirent légèrement la moyenne vers le haut.

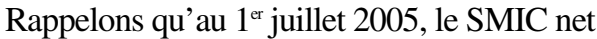
mensuel était de 958 euros.

La différence de salaire entre les hommes et les femmes est un résultat bien documenté dans la littérature (voir par exemple Meurs et Ponthieux, 2006) ${ }^{15}$. La situation relative des femmes en agriculture comparée à celle des hommes exerçant le même type d'emploi n'est pas pire que celle observée sur l'ensemble de la population active. Les ouvrières perçoivent un salaire inférieur de $17 \%$ à celui des ouvriers agricoles. En revanche, leur position absolue se situe vraiment en bas de l'échelle des revenus. En effet, on a vu que l'on pouvait rapprocher les salaires des ouvriers agricoles de ceux des employés du commerce, avec une position légèrement plus favorable pour ces derniers. En revanche, les salaires des hommes et des femmes employés de commerce sont proches (les femmes

15. Meurs D., Ponthieux S. (2006). L'écart des salaires entre les femmes et les hommes peut-il encore baisser ? Économie et Statistique, 398-399, p. 99-129.

Tableau 6. Distribution des salaires par secteur et qualification*

\begin{tabular}{l|c|c|c|c|c|c|c|c|c|c}
\hline & \multicolumn{2}{|c|}{ Tous secteurs } & \multicolumn{2}{c|}{ Agriculture } & \multicolumn{2}{c|}{ IAA } & \multicolumn{2}{c|}{ Autres industr. } & \multicolumn{2}{c}{ Commerce } \\
\cline { 2 - 12 } & total & ouvriers & total & ouvriers & total & ouvriers & total & ouvriers & total & empl. \\
\hline Salaire moyen & 1713 & 1342 & 1166 & 1064 & 1512 & 1318 & 1825 & 1405 & 1507 & 1177 \\
\hline $\begin{array}{l}\text { Médiane : } 50 \% \text { des } \\
\text { salariés ont moins de }\end{array}$ & 1483 & 1296 & 1100 & 1096 & 1322 & 1250 & 1522 & 1319 & 1278 & 1123 \\
\hline $\begin{array}{l}\text { 9e décile : } 90 \% \text { des } \\
\text { salariés ont moins de }\end{array}$ & 2700 & 1829 & 1617 & 1424 & 2269 & 1853 & 2906 & 1890 & 2362 & 1558 \\
\hline
\end{tabular}

Source : Enquête emploi 2005, traitements Inra

* Les effectifs des salariés agricoles présents dans l'enquête étant faibles et $80 \%$ des salariés de ce secteur étant sur des postes d'ouvriers, les qualifications ne peuvent être plus décontractées. 
gagnent en moyenne $4 \%$ de moins que leurs homologues masculins). Au total, ce sont bien les femmes ouvrières de l'agriculture qui occupent le bas de l'échelle des salaires.

\section{Quelques éléments de conclusion}

Lorsqu'on s'intéresse au travail agricole, on pense d'abord aux agriculteurs, à la maind'œuvre familiale et à la baisse continue de leurs effectifs. Cet état des lieux rapide met l'accent sur :

(i) le fait que près d'un tiers des heures travaillées sur les exploitations agricoles est effectué par des salariés ;

(ii) la diminution contenue des effectifs salariés agricoles en comparaison de celle enregistrée par la main-d'œuvre familiale ( $-5 \%$ contre $-18,2 \%$ entre 2000 et 2007 ).

D'un point de vue qualitatif, les emplois du secteur agricole sont des emplois essentiellement d'exécution, plus souvent précaires que dans les autres secteurs, occupés par des salariés peu qualifiés et faiblement rémunérés.

La place du salariat en agriculture, question peu étudiée, devient une question d'actualité (Peltier et al., 2009) ${ }^{16}$, notamment suite à la baisse du nombre d'exploitants et à l'agrandissement des structures des exploitations. Des réflexions prospectives

16. Peltier C., Marguet J., Privat C., Coulombel A. (2009). La place du travail salarié dans la gestion des exploitations agricoles. NESE, mars, p. 41-59. sont en cours au ministère de l'Agriculture, de l'Alimentation et de la Pêche, mais aussi à la MSA (MSA, 2009) ${ }^{17}$, sur le devenir du salariat agricole, sur les conditions d'attractivité du secteur, sur les actions à mener pour proposer des carrières et des perspectives aux salariés. Des questions de recherche, encore peu explorées, pourraient contribuer à éclairer cette réflexion. C'est le cas, par exemple, de la construction des parcours professionnels dans le secteur agricole : quels sont les enchaînements, à quoi conduisent les emplois saisonniers, quelles sont les conditions de stabilisation des salariés dans le secteur... Les pouvoirs publics soutiennent le développement des groupements d'employeurs, comme un outil permettant de faire coïncider l'offre et la demande de travail agricole, tout en favorisant une stabilisation des salariés dans le secteur et la construction de parcours professionnels pour des actifs peu mobiles. Il conviendrait donc d'étudier le rôle de ces structures au regard de ces objectifs. L'ensemble de ces questions ne peuvent être abordées sans considérer l'environnement économique et politique très fluctuant des exploitations agricoles, avec les variations fortes des niveaux de revenus (Blonde, Lesdos-Cauhapé, 2010) ${ }^{18}$, mais aussi dans la perspective de la PAC après 2013.
17. MSA (2009). Pour des emplois agricoles de qualité. Actes du colloque européen, 8 décembre 2008, Paris.

18. Blonde M. H., Lesdos-Cauhapé C. (2010). L'agriculture en 2009 en France et en Europe. Baisse des prix et des revenus. INSEE Première, $\mathrm{n}^{\circ} 1303$, juin. 


\section{LEXIQUE}

EARL - Exploitation agricole à responsabilité limitée. Forme de société civile spécifique à l'agriculture, régie par le Code rural, plus souple que le GAEC : la société unipersonnelle ou entre époux est admise, la participation de tous les associés aux travaux n'est pas obligatoire.

Exploitation agricole - $\mathrm{Au}$ sens de la statistique agricole, une unité économique de production de biens agricoles, qui dépasse 1 hectare de surface agricole utilisée (ou 20 ares de cultures spécialisées) et qui est soumise à une gestion unique.

Exploitations professionnelles - Elles ont une dimension économique de plus de 12 hectares de blé ou équivalent et emploient plus de 0,75 unité de travail annuel ou UTA.

GAEC - Groupement agricole d'exploitation en commun. Il se caractérise principalement par la participation de tous les associés aux travaux, une responsabilité financière limitée et le principe de transparence : les associés conservent sur le plan économique, social et fiscal leur statut de chef d'exploitation.

GE - Groupement d'employeurs. C'est une association loi 1901, constituée par des exploitations pour recruter un ou plusieurs salariés et les employer en commun. Le salarié du GE effectue des périodes de travail successives, sur la semaine, le mois ou l'année, auprès de chacune des exploitations adhérentes au groupement.

UTA - Unité de travail annuel, qui est un équivalent temps plein pour les enquêtes agricoles. Une UTA correspond au travail d'une personne à plein temps pendant une année entière.

SAU - Superficie agricole utilisée. Elle comprend les terres arables, la superficie toujours en herbe (STH) et les cultures permanentes. 